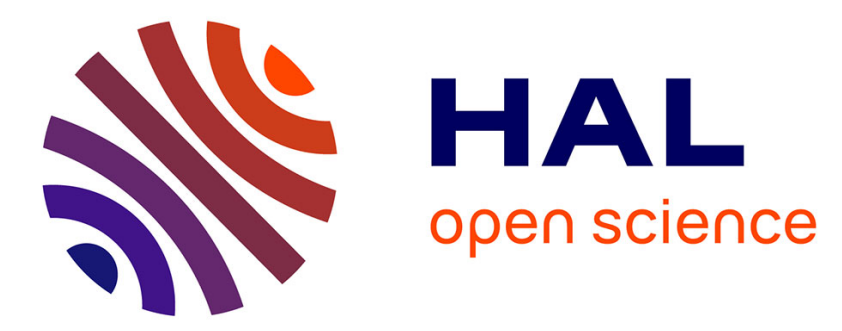

\title{
Finite element modeling of fluid-driven fracture in permeable medium
}

Benoît Carrier, Sylvie Granet

\section{To cite this version:}

Benoit Carrier, Sylvie Granet. Finite element modeling of fluid-driven fracture in permeable medium. Fifth Biot Conference on Poromechanics, Jul 2013, Vienna, Austria. pp. 462470., 10.1061/9780784412992.055-http://ascelibrary.org/doi/abs/10.1061/9780784412992.055 . hal00843753

\section{HAL Id: hal-00843753 \\ https://hal.science/hal-00843753}

Submitted on 12 Jul 2013

HAL is a multi-disciplinary open access archive for the deposit and dissemination of scientific research documents, whether they are published or not. The documents may come from teaching and research institutions in France or abroad, or from public or private research centers.
L'archive ouverte pluridisciplinaire HAL, est destinée au dépôt et à la diffusion de documents scientifiques de niveau recherche, publiés ou non, émanant des établissements d'enseignement et de recherche français ou étrangers, des laboratoires publics ou privés. 


\title{
Finite element modeling of fluid-driven fracture in permeable medium
}

\author{
B. Carrier ${ }^{1}$ and S. Granet ${ }^{2}$
}

${ }^{1}$ Université Paris-Est, Laboratoire Navier (UMR 8205), CNRS, ENPC, IFSTTAR, F-77455 Marne-la-Vallée; email: benoit.carrier@enpc.fr

${ }^{2}$ Laboratoire LaMSID, UMR EDF-CNRS, 1 avenue du Général de Gaulle, 92140 Clamart, France; email: sylvie.granet@edf.fr

\section{Abstract}

In this article, we present a numerical method to model the propagation of a fluid-driven fracture in a poroelastic medium in the finite element framework. We developed a zero-thickness finite element to model the fracture. The fracture propagation is governed by a cohesive zone model. The fluid flow within the crack is described by the lubrication equation and the fluid pressure in the fracture acts as an hydraulic and mechanical boundary condition on the lips of the crack. The bi-dimensional pressure diffusion equation and the equilibrium equation in the surrounding porous medium are fully solved numerically. We compare the results of our numerical model with asymptotic analytical solutions. Our numerical model captures very well the analytical solutions in all the asymptotic propagation regimes. In addition, our zero-thickness element method gives access to a finer description of the fluid diffusion in the porous medium and of its coupled mechanical response.

\section{Introduction}

Fluid-driven fractures in porous media play a significant role in many important geotechnical problems. For instance, hydraulic fracture is commonly 
used to stimulate the productivity of oil or gas wells. On the contrary, near the sites of underground storage of pollutants such as radioactive waste or carbon dioxide, the propagation of fractures under gas pressure has to be avoided. However, modeling fluid-driven fractures remains a challenging problem because it involves several coupled phenomena and a singularity at the fracture tip.

In this paper we present a finite element model to compute the propagation of a fluid-driven fracture and the hydro-mechanical evolution of the surrounding porous medium. We developed a zero thickness finite element to discretize the fracture [Carrier, 2012]. Several recent contributions adopted interface elements to model fluid-driven fractures in impermeable [Chen, 2009] and permeable [Lobao, 2010, Sarris, 2001] media. In this work we extend the use of interface elements to a large range of viscosities of the fluid which fills the crack and of permeabilities of the surrounding medium. Moreover, we compare the results of our numerical model with analytical models in all asymptotic regimes of propagation to demonstrate the ability of our model to capture the fracture propagation for a wide range of parameters.

\section{$2 \quad$ Finite element model}

We consider the propagation of a fluid-driven fracture in a saturated porous medium. The porous medium is assumed to be governed by the usual equations of poroelasticity [Coussy, 2004]. In the fracture, the fluid flow is described by the lubrication equation

$$
\frac{\partial \rho w}{\partial t}-\frac{\partial}{\partial x}\left(\frac{\rho w}{12 \mu} \frac{\partial p}{\partial x}\right)=0
$$

where $\rho$ is the fluid density, $w$ the fracture aperture, $\mu$ the fluid viscosity and $p_{f}$ the fluid pressure. The hydraulic fracture aperture is equal to the normal displacement jump across the fracture. A constraint of continuity of fluid pressure across the fracture is enforced. The fracture is filled with a fluid at pressure $p_{f}$ and we define the effective normal stress $\tau^{\prime}$ across the interface

$$
\tau_{n}^{\prime}=\tau_{n}+p_{f}
$$


where $\tau_{n}$ is the normal tension across the interface. The propagation of the fracture is governed by a cohesive zone model [Barenblatt, 1962, Dugdale, 1960]. The linear cohesive law used in this work is displayed on Figure 1-a. As long as the stress across the crack path is lower than the critical stress $\tau_{c}$, there is a perfect bonding. When the stress reaches $\tau_{c}$, the interface damages. During this stage, cohesive forces act against the opening stress. When the fracture energy $G_{c}$ has been dissipated, the crack lips are then stress free. Figure 1-b shows the stress profile along the interface.
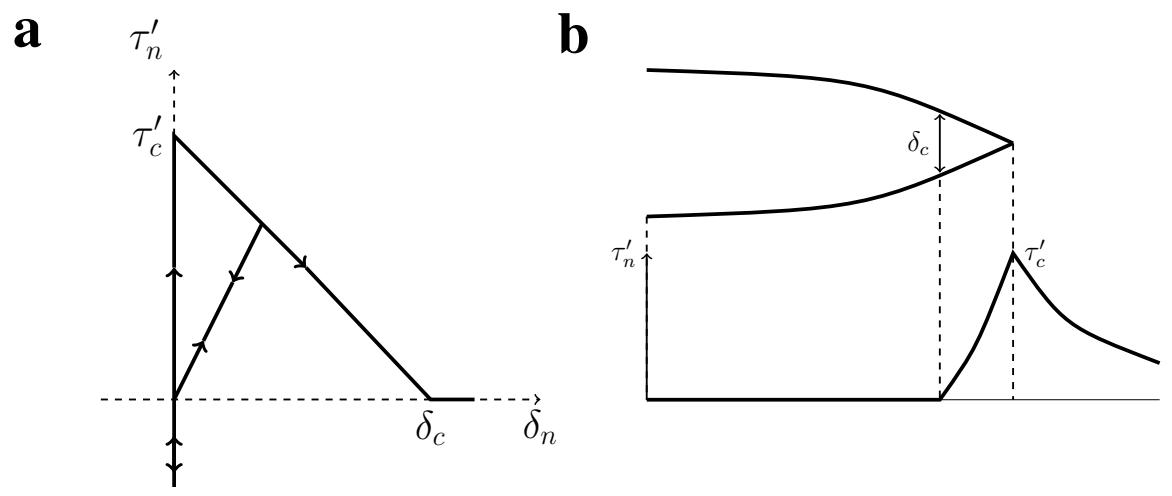

Figure 1: a) Linear cohesive law in the direction normal to the fracture. b) Fracture profile and effective stress along the fracture path.

To model the fracture propagation, we develop a zero-thickness cohesive element. This element can be easily incorporated in a finite element mesh along a postulated fracture path. This zero-thickness element is a degenerated quadrangle and is displayed in Figure 2. The element has both mechanical and hydraulic degrees of freedom. The outer segments (nodes 1,5,2 and $3,7,4)$ are linked to bulk P2/P1 hydro-mechanical elements. The middle segment carries the fluid pressure in the fracture degrees of freedom. $q$ on node 5 and 7 is a Lagrange multiplier to ensure the constraint of continuity of the pressure between the middle segment and the outer segments.

\section{Benchmark}

With our zero-thickness finite element method, we solve the KGD plane strain fracture problem [Khristianovic, 1955, Geertsma, 1969] (Figure 3). An incompressible fluid is injected at the rate $Q_{0}$ at the mouth of a fracture. 


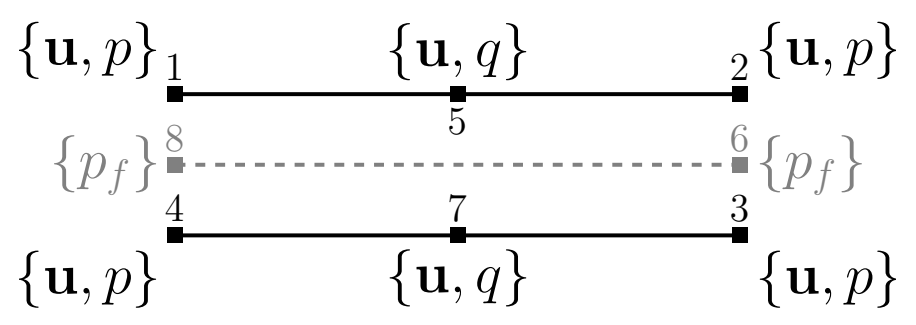

Figure 2: Zero-thickness element for fracture with hydro-mechanical coupling.

The surrounding medium is assumed to be elastic and infinite. Due to the symmetry of the problem, we consider only half of the space. The material parameters used in the numerical simulations are given in Table 1. The confining stress is always chosen large enough to ensure that the fluid lag at the fracture tip is equal to zero [Adachi, 2008].

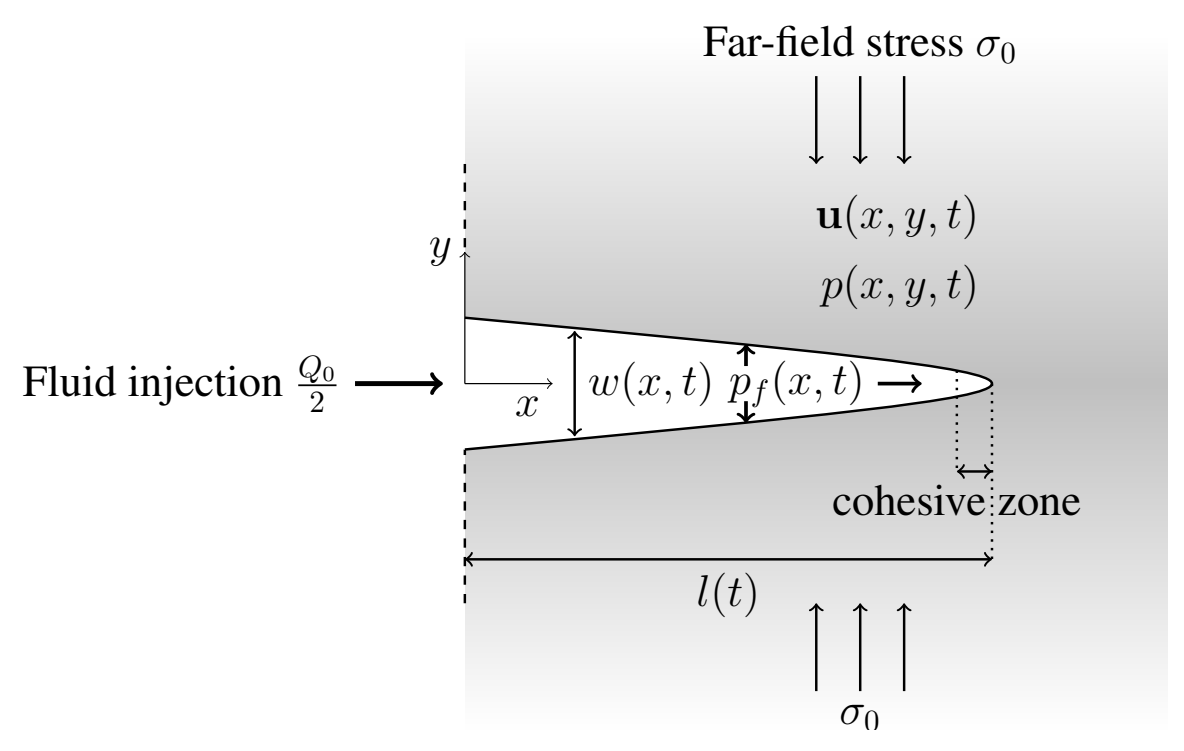

Figure 3: Propagation of an hydraulic fracture of length $l$ due to the injection of a viscous fluid at a rate $Q_{0} / 2$.

Figure 4 shows the fluid diffusion pattern around the fracture for various permeabilities. As expected, the higher the permeability is, the greater is the amount of fluid diffusing in the surrounding medium and the shorter is the fracture. 
Table 1: Material parameters

\begin{tabular}{c|c|c}
$Q_{0}$ & Injection rate & $0.001 \mathrm{~m}^{2} . \mathrm{s}^{-1}$ \\
$\sigma_{0}$ & Confining stress & $3.7 / 5.0 / 7.2 \mathrm{MPa}$ \\
\hline$E$ & Young's modulus & $17 \mathrm{GPa}$ \\
$\nu$ & Poisson's ratio & 0.2 \\
\hline$G_{c}$ & Fracturation energy & $120 \mathrm{~Pa} . \mathrm{m}$ \\
$\tau_{c}$ & Critical stress & $1.25 \mathrm{MPa}$ \\
\hline$M$ & Biot modulus & $68.7 \mathrm{MPa}$ \\
$b$ & Biot coefficient & 0.75 \\
$\Phi_{0}$ & Porosity & 0.2
\end{tabular}

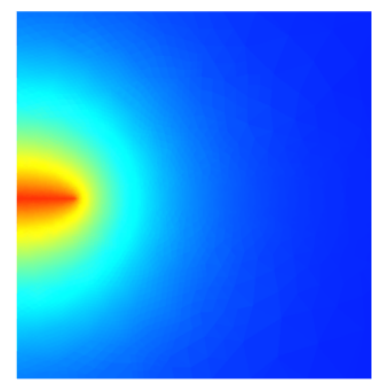

$\kappa=5 \cdot 10^{-15} \mathrm{~m}^{2}$

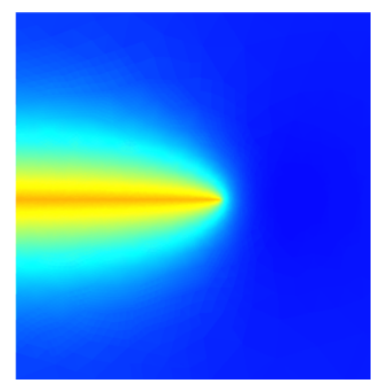

$\kappa=1.10^{-15} \mathrm{~m}^{2}$

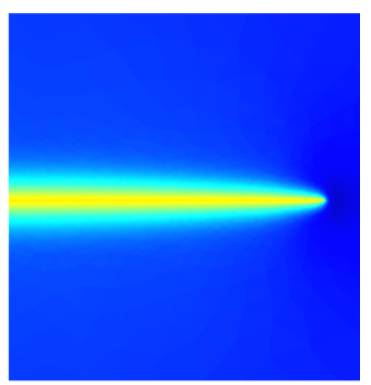

$\kappa=1.10^{-16} \mathrm{~m}^{2}$

$-1.12 e+06$

$2.94 \mathrm{e}+06$

$7 e+06$

Figure 4: Fluid pressure distributions (in Pa) at $t=30 \mathrm{~s}$ for $\mu=0.001 \mathrm{~Pa} . \mathrm{s}$ and for several permeabilities $\kappa$ of the porous medium. We show only the part of the mesh in the vicinity of the fracture. The side of each square pressure map is $18 \mathrm{~m}$ long.

In order to validate the numerical model, we compare the results of our simulations with the available analytical solutions of the KGD fracture problem. The analytical analysis of fluid-driven fractures in permeable media showed that during fracture propagation two energy dissipation processes and two fluid storage processes compete [Detournay, 2004, Adachi, 2008]. Energy can be dissipated either by fracturation or by fluid viscous flow. The two fluid storage processes are fluid storage in the fracture and fluid leak-off in the surrounding porous medium. When the energy dissipated by viscosity is negligible compared to the energy dissipated by fracturation, the fracture 
propagates in the toughness dominated regime. In contrast, it propagates in the viscosity dominated regime when viscosity is preponderant. In addition, at short times the fracture propagates in the storage dominated regime and evolves to the leak-off dominated regime at long times. The characteristic time of the evolution depends on the material parameters. By combining the dissipation and storage mechanisms, four asymptotic propagation regimes can be identified: Storage-toughness (so-called $K$-vertex regime), leak-offtoughness ( $\tilde{K}$-vertex regime), storage-viscosity ( $M$-vertex regime) and leakoff-viscosity ( $\tilde{M}$-vertex regime).

First, we choose a viscosity $\mu=10^{-4}$ Pa.s so that the fracture propagates in the toughness regime. Figure 5 displays the fracture length in function of time for a permeability $\kappa=10^{-16} \mathrm{~m}^{2}$. With this permeability, the characteristic time of leak-off is $\sim 10^{7} \mathrm{~s}$ and is orders of magnitude greater than the simulation time. Therefore, we compare the numerical fracture length with the analytical near- $K$ solution [Bunger, 2005] and note a very good agreement between the two solutions.

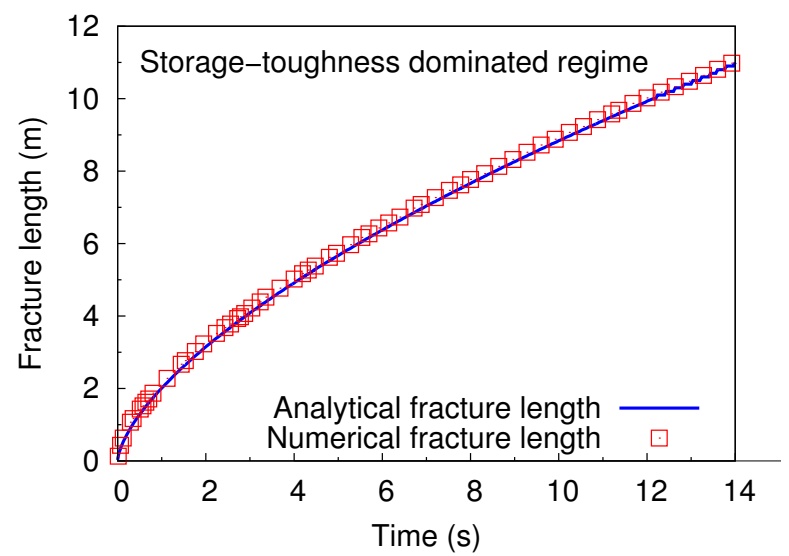

Figure 5: Fracture length evolution in the near- $K$ regime. The parameters are $\kappa=10^{-16} \mathrm{~m}^{2}, \mu=10^{-4} \mathrm{~Pa} . \mathrm{s}$ and $\sigma_{0}=3.7 \mathrm{MPa}$.

Figure 6 shows the fracture length in function of time for a permeability $\kappa=5 \times 10^{-15} \mathrm{~m}^{2}$. In that case, the characteristic time of leak-off is $\sim 1 \mathrm{~s}$ and the fracture propagation reaches very quickly the leak-off regime. We compare the results of the simulations with the analytical near- $\tilde{K}$ solution [Bunger, 2005]. In the analytical model, the fluid leak-off is assumed to be unidimensional. The monitoring of the leak-off during the numerical simu- 
lation shows that this assumption is not valid for the considered parameters and the computed fracture length is shorter than the prediction of the analysis solution. However, we show the result of another simulation in which we impose an unidimensional fluid diffusion. With 1D diffusion, the agreement between the numerical and the analytical solution is very good in the leak-off toughness regime.

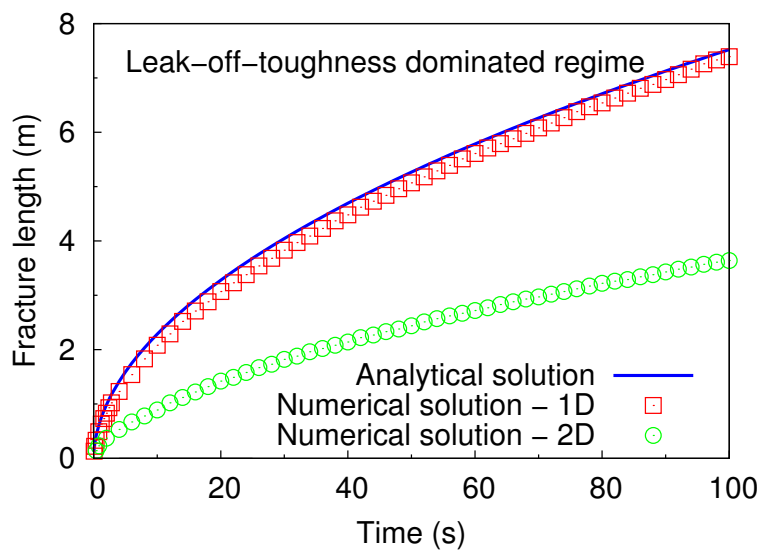

Figure 6: Fracture length evolution in the near- $\tilde{K}$ regime. The parameters are $\kappa=5 \times 10^{-15} \mathrm{~m}^{2}, \mu=10^{-4}$ Pa.s and $\sigma_{0}=5.0 \mathrm{MPa}$. We show both the results of a simulation in which the full bi-dimensional diffusion equation is solved in the surrounding medium and of a simulation in which the diffusion is constrained to be one dimensional.

For a viscosity $\mu=0.1$ Pa.s, the fracture propagates in the viscosity regime. Figure 7 displays the evolution in the fracture length for a permeability $\kappa=10^{-15} \mathrm{~m}^{2}$. For this choice of parameters, the fracture propagates in the storage-toughness regime and we compare our numerical results to the $M$ analytical solution [Adachi, 2008]. We note a small discrepancy between the analytical solution and the fully coupled numerical solution. The small fluid leak-off indeed induces a so-called back-stress [Vandamme, 1990], due to the increase of pore pressure in the vicinity of the fracture. This back-stress generates an increase of the injection pressure and of the fracture length and a decrease of the fracture aperture. When we remove the hydro-mechanical coupling in the porous medium by taking a Biot coefficient $b=0$ (uncoupled case on Figure 7), the discrepancy disappears.

We now increase the leak-off coefficient by increasing the permeability to 


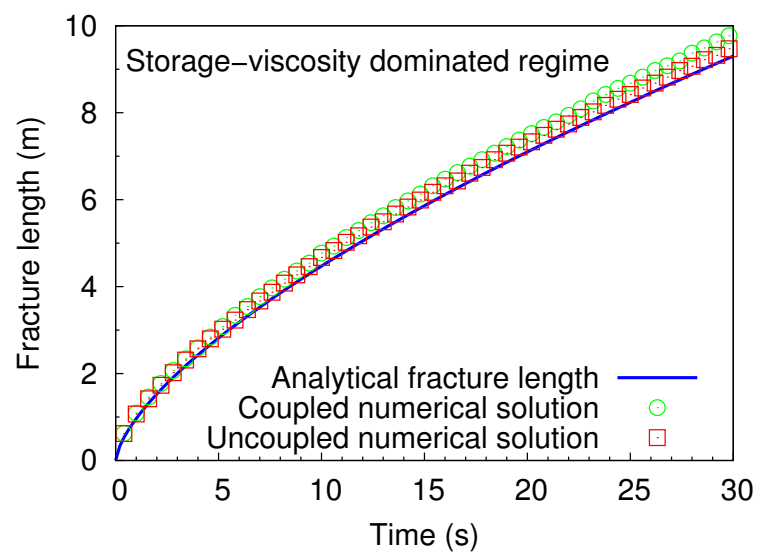

Figure 7: Fracture length evolution in the $M$ regime. The parameters are $\kappa=10^{-15} \mathrm{~m}^{2}, \mu=0.1$ Pa.s and $\sigma_{0}=3.7 \mathrm{MPa}$. We show both the results of a simulation in which the hydro-mechanical in the surrounding medium are fully coupled and of the results a simulation in which the hydro-mechanical coupling is removed by imposing a Biot coefficient equal to zero to remove any back-stress.

$\kappa=5 \times 10^{-12} \mathrm{~m}^{2}$. Figure 8 shows the analytical solution in the $\tilde{M}$ regime [Adachi, 2008] and the results of three simulations. The first simulation (diamonds on Figure 8) is the complete resolution of the coupled hydromechanical equations and of the 2D diffusion equation. In the two other simulations, we impose an unidimensional diffusion. The hydro-mechanical equations are coupled in the second simulation (circles) while they are uncoupled in the third (squares). The 1D diffusion uncoupled simulation is in very good agreement with the analytical solution. Both the back-stress and the diffusion pattern have indeed a crucial impact on the propagation of the crack.

\section{Conclusion}

In this work, we present a numerical method based on a zero-thickness finite element to model the propagation of a fluid-driven fracture. We compare the results of our numerical models with analytical solutions in the four asymptotic propagation regimes: Storage-toughness, leak-off-toughness, storage-viscosity and leak-off-viscosity. When we impose an unidimensional 


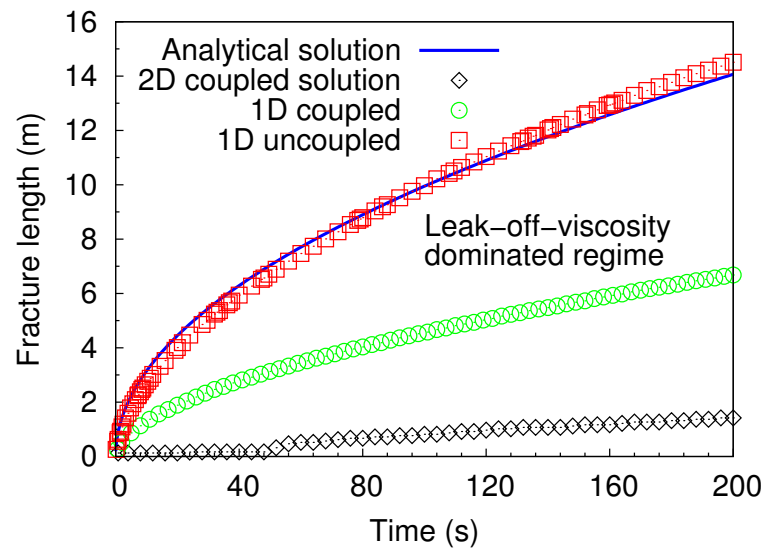

Figure 8: Fracture length evolution in the $\tilde{M}$ regime. The parameters are $\kappa=5 \times 10^{-12} \mathrm{~m}^{2}, \mu=0.1 \mathrm{~Pa} . \mathrm{s}$ and $\sigma_{0}=7.2 \mathrm{MPa}$. We show the results of three simulations with various assumptions regarding hydro-mechanical coupling and fluid diffusion in the surrounding medium.

leak-off and remove any back-stress from our numerical model, the numerical solutions are in very good agreement in all propagation regimes.

\section{References}

[Adachi, 2008] Adachi, J. and Detournay, E. (2008). Plane strain propagation of a hydraulic fracture in a permeable rock. Eng. Fract. Mech., 75(16):4666-4694.

[Barenblatt, 1962] Barenblatt, G. (1962). The mathematical theory of equilibrium cracks in brittle fracture. Adv. Appl. Mech., 7(55-129):55-130.

[Bunger, 2005] Bunger, A., Detournay, E., and Garagash, D. (2005). Toughness-dominated hydraulic fracture with leak-off. Int. J. Fract., 134(2):175-190.

[Carrier, 2012] Carrier, B. and Granet, S. (2012). Numerical modeling of hydraulic fracture problem in permeable medium using cohesive zone model. Engineering Fracture Mechanics, 79:312-328.

[Chen, 2009] Chen, Z., Bunger, A., Zhang, X., and Jeffrey, R. (2009). Cohesive zone finite element-based modeling of hydraulic fractures. Acta Mech. Solida Sinica, 22(5, Sp. Iss. SI):443-452. 
[Coussy, 2004] Coussy, O. (2004). Poromechanics. John Wiley \& Sons Inc. [Detournay, 2004] Detournay, E. (2004). Propagation regimes of fluid-driven fractures in impermeable rocks. Int. J. Geomech., 4:35-45.

[Dugdale, 1960] Dugdale, D. (1960). Yielding of steel sheets containing slits. J. Mech. Phys., 8(2):100-104.

[Geertsma, 1969] Geertsma, J. and De Klerk, F. (1969). A rapid method of predicting width and extent of hydraulically induced fractures. J. Pet. Technol., 21(12):1571-1581.

[Khristianovic, 1955] Khristianovic, S. and Zheltov, Y. (1955). Formation of vertical fractures by means of highly viscous fluids. In Proc. 4 th World Petroleum Congress, Rome, volume 2, pages 579-586.

[Lobao, 2010] Lobao, M., Eve, R., Owen, D., and de Souza N, E. A. (2010). Modelling of hydro-fracture flow in porous media. Eng. Comput., 27(12):129-154.

[Sarris, 2001] Sarris, E. and Papanastasiou, P. (2001). Modelling of hydraulic fracturing in a poroelastic cohesive formation. Int. J. Geomech.

[Vandamme, 1990] Vandamme, L. and Roegiers, J. (1990). Poroelasticity in hydraulic fracturing simulators. J. Pet. Technol., 42(9):1199-1203. 